Voix et Images

voixetimages

\title{
De Montréal à Bornéo
}

\section{Michel Biron}

Volume 22, numéro 3 (66), printemps 1997

\section{Gilbert Langevin}

URI : https://id.erudit.org/iderudit/201332ar

DOI : https://doi.org/10.7202/201332ar

Aller au sommaire du numéro

\section{Éditeur(s)}

Université du Québec à Montréal

\section{ISSN}

0318-9201 (imprimé)

1705-933X (numérique)

Découvrir la revue

\section{Citer cet article}

Biron, M. (1997). De Montréal à Bornéo. Voix et Images, 22(3), 619-623.

https://doi.org/10.7202/201332ar d'utilisation que vous pouvez consulter en ligne.

https://apropos.erudit.org/fr/usagers/politique-dutilisation/ 


\section{De Montréal à Bornéo}

\section{Michel Biron, Université du Québec à Montréal}

Je ne connais pas d'œuvre, dans la littérature québécoise, qui se range aussi expressément que le dernier livre de Régine Robin sous le signe tragique d'Auschwitz et de l'Histoire $^{1}$. Non pas sur le mode du souvenir ou du témoignage, à la façon bouleversante de Primo Levi ou, plus récemment, de Jorge Semprun, mais par le biais d'une méditation fragmentée sur les langues, sur les mots, sur les noms de lieux, de personnes, sur l'exil. La narratrice de L'immense fatigue des pierres incarne avec une conscience jamais apaisée la figure qui, selon Hannah Arendt, représente le plus fortement le $\mathrm{xx}^{\mathrm{e}}$ siècle: la personne déplacée ${ }^{2}$. Fatiguée de tant d'Histoire" (23), celle d'hier (Auschwitz) comme celle de jadis (Massada), elle cherche "un coin où l'Histoire cesse d'enfoncer ses vrilles dans la chair humaine, un coin d'ombre amnésique" (26). Elle n'ira pas se retirer dans la solitude du désert trop chargé d'Histoire lui aussi mais se rend au cour de l'Amérique, en pleine modernité: elle quitte son appartement de la rue du ChercheMidi pour habiter une tour à New York. Puis ce sera Montréal, villerefuge où l'on n'est jamais véritablement chez soi, mais, à cause justement de cela, lieu idéal d'écriture. Montréal offre à l'écrivain - moyennant quelques précautions pour ne pas s'engluer dans l'idéologie nationaliste - un espace dégagé de l'Histoire, un lieu qui soit à la fois un "nulle-part " (42) et un carrefour des cultures. Pas question de chercher à sortir du monde, comme l'archaïque héros de la modernité qui voulait s'en aller * anywhere out of the world ". Tout en voulant s'arrêter, ne plus être une "personne déplacée ", la narratrice choisit d'opposer le mouvement au mouvement, comme si plus de modernité pouvait venir à bout de la modernité elle-même (ce qui serait alors au sens propre la "postmodernité ", l'après-modernité), comme si elle pouvait habiter en même temps l'ici et l'ailleurs, l'Amérique et l'Europe.

Dans le texte d'ouverture, la narratrice s'appelle Nancy Nibor; ailleurs ce sera Pamela Wilkinson, Emilia Morgan, Martha Himmelfarb ou Régine Robin tout simplement, selon une identification à laquelle tout invite, depuis le palindrome du début (Nibor) jusqu'à cette étrange désignation générique que sont les abiofictions". Mais les identités, à l'heure de l'immatériel, du virtuel et de la biotechnologie, ça bouge sans arrêt et il vaut mieux ne pas trop se fier aux noms propres. Nancy Nibor, donc, puisqu'il faut bien l'appeler par quelque nom, est une romancière américaine à succès dont la fille s'est installée à Jérusalem après la guerre des Six-Jours. Elles se sont éloignées l'une de l'autre et se rencontrent depuis au hasard de leurs déplacements, surtout dans les aéroports. Les destins respectifs de la mère et de la fille sont présentés en alternance, jusqu'à ce que la narratrice leur coupe à toutes deux la parole et s'adresse directement au lecteur pour 
lui expliquer deux ou trois vérités élémentaires et prévenir en particulier toute tentation de confondre l'auteur et la narratrice: ‘J'ai vieilli la mère. Par rapport à moi, évidemment. Elle a plus de soixante ans. Cela écartera les malentendus avec vous lecteur et les tentations de projections chez moi " (37). Ces apartés, loin de soutenir le récit, freinent, détournent sa progression, comme si la narratrice, sentant des cailloux sous ses pieds (des scrupules au sens étymologique), devait s'arrêter de temps à autre pour secouer ses souliers.

Mais il est vrai qu'ici la notion même de récit ne va pas de soi. Alors que des textes comme - Le dibbouk inconnu ", :Gratok. Langue de vie et langue de mort * et - Mère perdue sur le World Wide Web " comportent indéniablement une dimension narrative prépondérante, les autres *biofictions" se structurent autour de formes telles que l'agenda (.L'agenda), le journal ou le courrier électronique ("Journal de déglingue entre le Select et Compuserve") et le carnet de citations ou la liste généalogique (*Manhattan Bistrow). Le texte final conclut, avec une gravité extrême, à l'impossibilité du récit: «Une Histoire qui ne peut être narrée, racontée, mise en mots. / Des listes à établir. / Pas de récit. * (160) Le récit serait en quelque sorte immoral, la négation de .l'immense fatigue des pierres " qui est le motif principal de ces textes. Comment ne pas songer en lisant ceci à la fameuse sentence d'Adorno, selon laquelle e écrire un poème après Auschwitz est barbare.?

Reste que nous ne sommes plus dans l'immédiat après-coup d'Auschwitz et que le récit resurgit de la frag- mentation même des formes et de la confusion des identités. Il me semble qu'une moitié du livre donne tort à l'autre moitié et trahit une sorte de nécessité du récit. Peut-être même s'agit-il de la moitié la plus forte, celle qui donne sa couleur à l'ensemble des textes. Le récit, en tant que fiction, a plus qu'une fonction supplétive: le fictif s'empare du réel et le met à sa main. Dans "Le dibbouk inconnu ", l'une des ibiofictions " où la forme et le sujet s'intègrent le plus fortement l'un à l'autre, le narrateur, Michel Himmelfarb, se rend à Washington pour visiter le musée de l'holocauste. À l'entrée, il doit choisir une carte d'identité portant le nom d'une victime de l'holocauste. Pour l'heure, Michel Himmelfarb s'appelle David Morgensztern. L'enfant qui portait ce nom, constate avec stupéfaction le narrateur en lisant sa carte d'identité, est né dans la même ville que lui en Pologne. Il est donc tout à fait possible qu'il ait réellement rencontré (voire fréquenté) ce gamin avant de quitter la Pologne, à l'âge de sept ans. Comme tous les narrateurs du livre en pareilles circonstances, celui-ci va réfléchir à cet événement quelque part où il puisse fumer ses Saint Moritz Menthol et boire du whisky. Puis il rentre chez lui, à Paris, où il fera de même. Sans penser à sa compagne Danièle, qu'il adore par ailleurs, il se lance de toute urgence dans son réseau de convivialité Internet où il s'était déjà inventé une biographie, celle de sa sœur morte (Rivka) à Treblinka, à qui il voulait ainsi conserver une sorte d'existence posthume. Cette fois, cependant, il change de personnage : il sera David Morgensztern, son possible camarade d'enfance. L'histoire de ce dernier, sa "biofic- 
tion ", emporte un succès instantané sur le réseau. Après quelque temps toutefois, un homme lui téléphone pour lui demander s'il se nomme bel et bien David Morgensztern. Il s'agit, comme on le devine, du vrai David Morgensztern (enfin, qui se prétend tel), lequel raconte son histoire, passablement différente de celle que le narrateur s'était inventée. D'où sa colère et finalement sa décision de maintenir sa fiction en dépit du soidisant authentique David. Mais alors, quelqu'un se présente sur le réseau sous son nom à lui, Michel Himmelfarb, et le narrateur (tout à la fois Michel, David et Rivka) sent le sol se dérober sous ses pieds, pas trop longtemps, jusqu'à ce qu'il retrouve ses sens et conclue sereinement à la revanche de la littérature sur la biologie.

Toute la fin de cette "biofiction" se déroule à vitesse supérieure, comme si le texte courait vers sa conclusion sans se préoccuper de développer l'intrigue, une fois nouée. Le récit s'interrompt brusquement au moment même où les choses prennent de l'épaisseur, où se crée l'attente du lecteur. C'est aussi le cas, par exemple, de l'extraordinaire quête d'une "Mère perdue sur le World Wide Web", qui se termine abruptement. Une telle discontinuité est sans doute la loi du genre: chaque "biofiction" se structure autour d'un ou de plusieurs déplacements d'identité sans pour autant qu'il y ait enchaînement des péripéties. Ce peut être un simple jeu, comme lorsque la diariste du .Journal de déglingue entre le Select et le Compuserve" se fait passer pour une personne qu'elle n'est pas. Ce peut être aussi un métier, comme c'est le cas pour le même personnage, propriétaire d'une boutique montréalaise qui vend des "biographies sur mesure. Il va sans dire que de telles biographies n'ont rien à voir avec les récits biographiques traditionnels: si le sujet est de retour, c'est un sujet à la fois dépossédé de lui-même et libéré du poids de son passé, immergé dans un monde où les noms sont devenus interchangeables, où une mère ne reconnaît plus ses petits.

\section{$*$}

De l'action imprévisible, des péripéties, il n'en manque pas dans le dernier roman de Gilles Marcotte, Une mission difficile ${ }^{3}$. Le premier réflexe du lecteur, devant ce drôle de petit objet recouvert de la chevelure jaune d'une femme aux allures d'espionne ${ }^{4}$, est de se demander s'il ne s'est pas trompé d'enveloppe, s'il n'est pas sur le point d'ouvrir un roman d'action de série B signé par un Gilles Marcotte qui, si l'on appliquait la leçon de la "biofiction", pourrait bien être un autre que le critique littéraire que l'on connaît. Les premières lignes auront vite fait de le détromper - et de chasser illico tout amateur de série $B$. Nous voici à l'intérieur de l'édifice qui abrite l'Organisation au service de laquelle travaille le narrateur. L'architecture en est des plus curieuses: au centre se trouve une grande pièce ronde au tapis très bleu où convergent tous les couloirs menant aux bureaux. Pour aller d'un couloir à l'autre, il faut donc obligatoirement passer par cette pièce étrangement vaste et cruellement bleue où tout commence et recommence. Oubliez la forme linéaire, 
progressive du roman d'action moderne: tout changement de direction (de couloir) suppose un retour au point d'origine, au centre du Centre. Et qu'y a-t-il précisément dans ce lieu hautement symbolique? Une femme. Sa fonction au sein de l'Organisation demeure vague. Tout ce qu'on sait, c'est qu'elle règne comme une âme au milieu de cet espace creux, ouvert à toutes les projections.

Du vide au plein, de la rotonde bleue à la jungle la plus verte, il n'y a qu'un simple changement de décor. - Me voici donc à Bornéo ", lance le narrateur au moyen d'un .donc. qui se passe de commentaires. De même, Bornéo se passe de description (pour cela, prière, nous dit-on, de consulter le National Geographic ou de visionner quelque film documentaire). On saute tout de suite dans le vif de l'action, enfin presque, car il faut préalablement assurer la communication avec le Centre, c'est-à-dire avec la femme. Le héros envoie le * télégramme d'usage ", mais il ne se contente pas de donner signe de vie: il profite de l'occasion pour citer la figure de la cantatrice Joan Sutherland entendue à Singapour, puis compare celle-ci à sa destinataire, etc. On devine la réponse du Centre: -FRAIS DE TÉLÉGRAMME EXCESSIFS STOP . À quoi on ajoute, comme si les considérations matérielles ne préoccupaient pas beaucoup plus l'Organisation que le héros: *SOUVENEZ-VOUS D'OSTENDE * (16). Nous irons donc à Ostende, le temps que le héros se remémore une mission périlleuse au cours de laquelle il a bien failli laisser sa peau, à cause d'une femme bien sûr.

De retour à Bornéo, le héros, assisté par un guide-interprète dayak très au fait de la littérature occidentale, se dirige vers l'objet de tous ses désirs, non sans avoir saucissonné un serpent chemin faisant. Il arrive au terme de sa mission, malade mais en vie, et que voit-il? Une idole poilue assez repoussante, qu'il se met à démolir furieusement à coups de pioche. À la fin, il ne reste presque plus rien de la chose, que des cailloux difformes, d'où émanent pourtant encore des voix, des chants, comme si l'idole, même détruite, continuait à obséder le héros. Changement de décor: le deuxième chapitre se passe à Grand Rapids, Michigan, chez le frère du narrateur qui est deuxième trompette dans l'Orchestre municipal. Il n'y a plus de serpent ni d'idole, mais les dangers ne sont pas moins mortels à Grand Rapids qu'à Bornéo. La preuve, c'est que le héros se trouve devant une femme qui le menace d'un revolver, sans que l'on sache pourquoi. Il est vrai qu'on ne se retire pas impunément de l'Organisation, comme il a tenté de le faire en se réfugiant chez son frère. Mais les causes exactes importent peu en l'occurrence - pas besoin de pratiquer la psychanalyse pour comprendre la logique de telles angoisses ("Elle me vise le sexe, ma parole!", 53). Imaginez la scène: la femme, une cantatrice renommée internationalement, est née à Saint-Lin (Québec). Après une carrière exceptionnelle sur les plus grandes scènes du monde, elle s'est engagée dans l'Organisation, avec pour seul regret de n'avoir jamais interprété le rôle d'Isolde. Qu'à cela ne tienne: la tueuse à gages et notre héros chanteront ensemble les grands airs de Tristan et Isolde. Après quoi, les choses se compliquent rapidement: le frère surgit, entre dans la chambre 
où se trouvent les amoureux éternels et se fait liquider par celle * qui s'appelle peut-être Olga " (65), etc.

De retour encore une fois à Bornéo, le héros n'a plus de mission précise, mais les buts exacts ne tiennent pas plus de place dans sa destinée que les causes. Fini toutefois le plaisir de la découverte: la jungle, c'est du déjà vu. Même le sorcier dayak n'a plus rien du sorcier qu'il était la première fois, comme si le contact de la civilisation l'avait purgé de ses pouvoirs spirituels et réduit à n'être qu'un homme de prose, au langage particulièrement vulgaire. Soudain, le héros tombe sur le Grand Patron lui-même de l'Organisation, parti à sa recherche à la demande expresse de la femme. Avant même que ce patron dévoué, bon père de famille et professeur à l'Université d'Ottawa, n'ait fini d'expliquer sa présence en pleine jungle, une flèche empoisonnée le tue sur-le-champ. Sans hésiter, le héros s'en va transmettre à son Centre la nouvelle de l'accident, mais notre prince du télégramme, tout à la poésie du médium, en oublie le message initial, ce qui donne finalement (après deux essais brûlés) ce texte savoureux:

DANS CETTE FORÊT OBSCURE STOP JE PEINE À L'INFINI STOP VIVANTS PILIERS CONFUSES PAROLES STOP TU PARLES STOP VASE DE TRISTESSE STOP GRANDE TACITURNE STOP BELLE TÉNÉBREUSE STOP ET ENCORE SEMPER EADEM STOP OUBLIEZ-MOI STOP JE VOUS ORDONNE DE M'OUBLIER STOP JE DÉMISSIONNE STOP JE SUIS EN VILLÉGIATURE STOP INTROUVABLE STOP JE M'AMUSE BEAUCOUP STOP SED NON SATIATA STOP (86)
Toujours à Bornéo, le héros rencontre cette fois son ami Georges Papineau, ancien ambassadeur du Canada à Rio qui a décidé de tout abandonner pour écrire en paix, en pleine nature. À moins de s'appeler Paul Claudel, il est assez difficile, en effet, de concilier l'écriture et la diplomatie. L'écrivain, comme le sorcier dayak, a donc changé radicalement de monde, de langage. Auparavant, il incarnait, aux yeux du narrateur, - l'écrivain parfait, celui dont la foi dans la littérature ne s'embarrassait d'aucune confirmation extérieure . (43). À présent, le voici qui, sous prétexte de se donner enfin à la passion de l'écriture, n'écrit plus -que des récits de voyages. La vraie vie. Les dangers réels. Les instincts fondamentaux. Les adaptations pour la télévision. Les traductions. Le Book for the Month Club. (93). Dans la jungle, il ne manque de rien: muni de son ordinateur et de son télécopieur, il communique directement avec qui il veut. Il avoue même, candide: -Je ne savais pas que c'était aussi agréable d'être écrivain. (94). On aura compris, au terme de ce conte philosophique plein d'une ironie toute voltairienne, qu'Une mission difficile s'écrit précisément contre tout cela.

1. Régine Robin, L'immense fatigue des pierres. Biofictions, Montréal, XYZ éditeur, coll. - Étoiles variables -, 1996, 189 p.

2. Citée par Alain Finkielkraut, L'bumanité perdue. Essai sur le $\mathrm{xx}^{e}$ siècle, Paris, Seuil, 1996 , p. $145-146$.

3. Gilles Marcotte, Une mission difficile, Montréal, Boréal, 1997, 102 p.

4. Il s'agit de -M-Maybe (A Girl's picture). de Roy Lichtenstein, maître du pop-art. 